\title{
Modulation recognition for real HF signals
}

\author{
James E. Giesbrecht ${ }^{a, b, c}$, Russell Clarke ${ }^{c}$, and Derek Abbott ${ }^{a, b}$ \\ ${ }^{a}$ Centre for Biomedical Engineering, The University of Adelaide, SA 5005, Australia \\ ${ }^{b}$ School of Electrical \& Electronic Engineering, The University of Adelaide, SA 5005, Australia \\ ${ }^{c}$ Ebor Computing, 147 Henley Beach Rd., Mile End, SA 5031, Australia
}

\begin{abstract}
High-frequency (HF) communications is undergoing resurgence despite advances in long-range satellite communication systems. Defense agencies are using the HF spectrum for backup communications, as well as for spectrum surveillance applications. Spectrum management organizations are monitoring the HF spectrum to control and enforce licensing. These activities usually require systems capable of determining the location of a source of transmissions, separating valid signals from interference and noise, and recognizing signal modulation. Our ultimate aim is to develop robust modulation recognition algorithms for real HF signals, that is, signals propagating by multiple ionospheric modes with cochannel signals and non-Gaussian noise.

One aspect of modulation recognition is the extraction of signal identifying features. This paper continues our work of applying various feature parameters to real HF signals and gives guidance on which features show potential for use in robust recognition of HF modulation types in the presence of HF noise and multi-path. It also defines a measure of mean separation distance between modulation types based on an entropy parameter, and discusses the probability density function of HF noise.
\end{abstract}

Keywords: HF, HF Noise, Modulation-Recognition, Signal Features, Entropy, Coherence, Real Signals

\section{INTRODUCTION}

Long distance radio-communications became practical in the early-1900s with the experiments and inventions of Guglielmo Marconi. ${ }^{1}$ Marconi was able to demonstrate long-distance wireless communications because ionized gases in the ionosphere cause refraction of high-frequency (HF) signals (nominally $2 \mathrm{MHz}$ to $30 \mathrm{MHz}$ ).

Such refraction enables signals to propagate beyond the horizon to distant receivers unable to be reached by higher frequency (VHF and above) signals. This fact makes the HF band attractive for private and commercial interests as well as for defense forces spread across the globe. For example, spectrum management agencies monitor the HF band for unlicensed operators and military agencies use the HF band for communications.

A conventional HF receiver is usually constructed in a super-heterodyne configuration ${ }^{2}$ and consists of filters, oscillators, and many discrete components. Its function is to down-convert signals acquired by an antenna to baseband and to then demodulate the signal to extract the information content. A particularly versatile HF receiver may even have more than one demodulator so that signals of various types can be handled.

Today, there are so many different digital and analog modulation techniques that even the "versatile" receiver cannot be used for more than a few modulations. Many of these are of the family of space-time layered signals including spread spectrum, time-domain multiplexing (TDM), frequency-domain multiplexing (FDM), and parallel transmission of data through multiple antennas and/or frequencies. Traditional HF monitoring, processing, and analysis cannot easily handle these signals.

Software radio aims to replicate hardware functions in software running on a generic platform. In so doing many of the problems associated with receiver implementations are avoided. In addition, receiver chains can easily be changed to accommodate various modulation schemes. The idea is to digitize the incoming radiofrequency $(\mathrm{RF})$ signal directly and then to perform down-conversion and demodulation in digital hardware. This

Send correspondence to:

The University of Adelaide - E-mail: james@eleceng.adelaide.edu.au; Phone: +61 (8) 83036296

Ebor Computing - E-mail: james.giesbrecht@ebor.com; Phone: +61 (8) 8238 0300; Fax: +61 (8) 82380304

Microelectronics: Design, Technology, and Packaging II, edited by Alex J. Hariz,

Proc. of SPIE Vol. 6035, 60351S, (2006) · 0277-786X/06/\$15 · doi: 10.1117/12.638553

Proc. of SPIE Vol. $603560351 S-1$ 
is now possible with current high-resolution Analog-to-Digital Converters (ADCs) and Digital Down-Converters (DDCs).

This paper continues the work presented earlier by Giesbrecht, Clarke, and Abbott. ${ }^{3-6}$ In the context of the previous work, it briefly reviews the performance of the coherence function and discusses the outcome of applying Benedetto's entropy ${ }^{7}$ measure. This paper also discusses the non-Gaussian nature of electro-magnetic noise in the HF spectrum, and describes a way forward in the development of a robust modulation recognition system.

\subsection{Nomenclature}

This paper applies the following adjectives: real, which refers and applies to measurable HF processes or transmissions propagating by multiple ionospheric modes or ground waves; synthetic, which refers and applies to artificially contrived (e.g. by computer) HF processes or transmissions.

\section{MODULATION RECOGNITION}

The process of determining the modulation type of a signal with no foreknowledge of the signal modulation characteristics is known as modulation recognition. This field has been a topic of active research for the past 25 years. During those early days, modulation recognition was accomplished through multiple hardware demodulators - one for each modulation type of interest. With the advent of software radio, these multiple demodulators are being combined in software. With either method, the purpose of modulation recognition is to determine the type of modulation so that the correct demodulator can be chosen to demodulate the signal.

Fundamental processes of modulation recognition are parameter extraction, feature selection and classification. Parameter extraction determines unique characteristics of the signal the most basic of which are frequency, phase, and amplitude. Parameters may also include statistical measures such as standard deviation, and $m^{\text {th }}$ order moments and cumulants (e.g. kurtosis is a $4^{\text {th }}$ order cumulant). Typically these parameters form an $N$ dimensional vector. Feature selection transforms the $N$ parameters into an $M$ dimensional feature space where $M \leq N$. Ideally, the feature space provides $M$ orthogonal basis vectors which enable a classifier to separate signal types. Sometimes parameter extraction and feature selection are combined and called feature extraction. Classification associates features with modulation types. These associations are normally made through statistical methods, decision theoretic methods, artificial neural networks (ANNs), or pattern recognition algorithms. For the interested reader there are many useful references describing the general process of feature extraction and classification. $^{8-12}$ Classification is a vast area of research. Developing a robust classification algorithm is not an immediate aim of this research. The initial goal is to develop a robust method for feature extraction, the outputs of which can be applied to various classification algorithms.

\section{REVIEW OF RESEARCH}

Normally at this point in a paper a review of literature is warranted. However, this shall be deferred to later in the discussion for reasons that will soon become clear. The only review now necessary is that of previous work ${ }^{3-6}$ on coherence and Benedetto's entropy ${ }^{7}$ measure. Coherence is unlikely to yield good separation between signal types, but Benedetto's entropy parameter is a feasible indicator of signal type.

\subsection{Coherence}

In general, the coherence function is analogous to correlation coefficients in the frequency domain. Consider the power-spectral density of a signal $X$. It is defined as

$$
P_{x x}(f)=\int_{-\infty}^{\infty} \rho_{x x}(\tau) e^{-j 2 \pi f \tau} d \tau
$$

where $f$ is frequency, $\tau$ is time delay, and $\rho_{x x}(\tau)$ is the auto-correlation function of $X$. In a similar manner, the cross-spectral density is defined by

$$
P_{x y}(f)=\int_{-\infty}^{\infty} \rho_{x y}(\tau) e^{-j 2 \pi f \tau} d \tau
$$


where $\rho_{x y}(\tau)$ is the cross-correlation of signals $X$ and $Y$. From these definitions the coherence function is the cross-power spectral density normalized by the respective auto-power spectral densities;

$$
C_{x y}(f)=\frac{\left|P_{x y}(f)\right|^{2}}{P_{x x}(f) P_{y y}(f)} .
$$

For each frequency, the coherence function varies between 0 and 1 and indicates the similarity between $X$ and $Y$. If the value of the function at a particular frequency is close to unity, it indicates that $X$ and $Y$ are similar at that frequency. On the other hand, if the value of the function is near zero it implies that the two signals are dissimilar at the particular frequency. Note also, that this definition of coherence is unaffected by the phase of $X$ and $Y$ since $P_{x x}(f)$ and $P_{y y}(f)$ are real.

Coherence is unaffected by phase but it is affected by the message in some signals (e.g. FSK). Moreover, though the spectra of two signals may appear strikingly similar, it does not mean that the coherence will be high, especially in light of the message dependence. This effect is simulated and verified with real signals in previous work ${ }^{6}$ that shows coherence is impractical as a parameter for separation of real HF signals. At best, using coherence to identify an unknown signal is a brute-force method suitable only for signifying whether or not an unknown signal with unknown message is the same as a known signal with a known message.

\subsection{Entropy}

Benedetto, Caglioti, and Loreto $^{7}$ present a method whereby the similarity or dissimilarity of two information sources, $\boldsymbol{A}$ and $\boldsymbol{B}$, is defined by their relative entropy. The method compresses a long sequence $A$ from $\boldsymbol{A}$ and subtracts the compressed length from the length of the compressed sequence $A+b$, where $A+b$ is the concatenation of $A$ and a small sequence $b$ from $\boldsymbol{B}$. This is defined as the entropy of $\boldsymbol{A}$ (designated by $\Delta_{A b}$ ). In a similar manner they compute the entropy of $\boldsymbol{B}$ as $\Delta_{B b}$. They then define the relative entropy between $A$ and $B$ as

$$
S_{A B}=\frac{\Delta_{A b}-\Delta_{B b}}{|b|},
$$

where $|b|$ is the length of $b$, and the relative entropy between $B$ and $A$ as

$$
S_{B A}=\frac{\Delta_{B a}-\Delta_{A a}}{|a|},
$$

where $|a|$ is the length of a small sequence of $A$. The total entropy, or entropic distance, between the two information sources is then by definition

$$
S_{T} \equiv \frac{\Delta_{A b}-\Delta_{B b}}{\Delta_{B b}}+\frac{\Delta_{B a}-\Delta_{A a}}{\Delta_{A a}}=\frac{|b| S_{A B}}{\Delta_{B b}}+\frac{|a| S_{B A}}{\Delta_{A a}} .
$$

Previous investigations ${ }^{4,5}$ show that a change in received signal strength can be detected as a change in entropic distance and that it may be possible to identify a signal modulation with entropic distance.

\section{RESEARCH PLATFORM}

An HF Monitoring System and research platform is currently under development at Ebor Computing. This monitoring system consists of an array of antennas, followed by signal conditioning electronics, multiple digital receivers, and a data processing sub-system (see Figure 1).

The Analog RF Subsystem consists of antennas, amplifiers, attenuators, and filters. Outputs of this subsystem are fed to a rack of digital receivers that directly sample RF signals up to $50 \mathrm{MHz}$ and down-convert the $\mathrm{HF}$ channels to baseband. The digitization of the RF signals is achieved by high-speed analog-to-digital converters (ADCs). Down-conversion is accomplished with digital-down-converters (DDCs). The Data Processing Subsystem collects and analyzes the baseband information. A Remote Management System oversees the operation of the entire system. The Research Platform supports this work and operates independently of the Data Processing Subsystem. It comprises hardware and software tools as well as a remotely-controlled test-transmitter with HF modem (not shown) that transmits groundwave signals to the HF Monitoring System. 


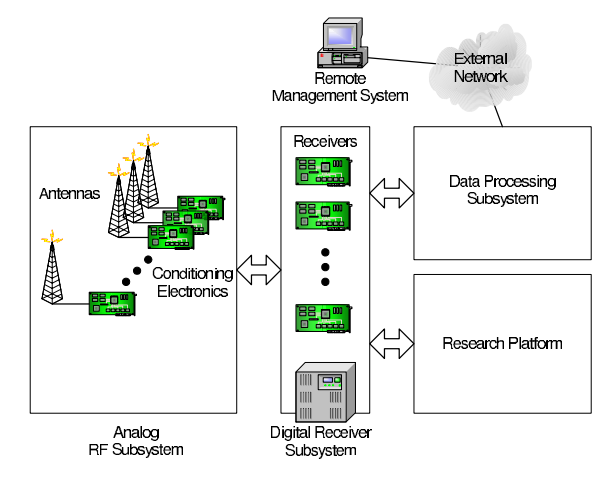

Figure 1. Architecture of the HF monitoring system.

\section{DATA COLLECTION}

\subsection{HF Signals}

An HF modem (British Aerospace ARM-9401) was setup with a HF transmitter (1 W) approximately $300 \mathrm{~m}$ from the antenna array of the HF Monitoring System. At this distance, the HF Monitoring System was able to receive groundwaves from the transmitter that were relatively unattenuated and undistorted but still containing noise and possibly interfering signals. The modem is capable of processing a number of different modulation schemes, but only four were considered in this work.

In turn, each of the four different modulation schemes (Types I to IV in Table 1) were transmitted by the HF modem on $15.824 \mathrm{MHz}$ USB (upper side-band). The research platform applied a $3 \mathrm{kHz}$ low-pass filter on the received signal since channels in the $\mathrm{HF}$ spectrum are generally $3 \mathrm{kHz}$ wide and the chosen modulations have bandwidths of $3 \mathrm{kHz}$.

\subsection{HF Noise}

To determine the background noise in the HF spectrum, a pre-emptive null-steering ${ }^{13}$ algorithm was used. In this method two digital down-converters (DDC) were swept across the HF band at $125 \mathrm{kHz} / \mathrm{s}$ from $2 \mathrm{MHz}$ to $25 \mathrm{MHz}$. The period of this sweep was 15 minutes. Each DDC had a bandwidth of approximately $4 \mathrm{kHz}$ and a sampling rate of approximately $10 \mathrm{kHz}^{*}$.

Sweeps were made at a "noisy" HF site over a 48-hour time span (February 16 - 17, 2005) in Adelaide, Australia. Only data sets created shortly before sunrise ${ }^{\dagger}$ and shortly after sunrise were considered for the purposes of the noise density measurement.

At sunrise the D-region in the ionosphere begins to form and to absorb distant man-made and natural interference propagating by ionospheric modes. Moreover, the level of local man-made interference is relatively low because people are still asleep. However, local man-made interference increases significantly as people awake and attend to their daily activities. Consequently, the "best" estimation of the HF noise distribution is possible during the sunrise period.

\section{METHODS}

The focus now turns to the application of total entropy (entropic distance) to real signals and a demonstration that the HF background noise plus interference appears to follow a Bi-Kappa distribution.

\footnotetext{
${ }^{*}$ The exact sampling rate was $\frac{31,250}{3} \mathrm{~Hz}$.

${ }^{\dagger}$ On February 17, 2005 sunrise was at 6:51 am in Adelaide, Australia.
} 


\subsection{Measurement of Entropy}

For the measurement of entropy, the information source (or unknown received signal) $\boldsymbol{A}$ consists of a baseband HF signal; the reference signal, $\boldsymbol{B}$, is a continuously random selection of symbols from a uniformly distributed alphabet. The idea then, is to determine the entropic distance between $\boldsymbol{A}$ and $\boldsymbol{B}$ for the signal types in Table 1 and in Figure 2.

Table 1. Various HF signal types used for entropy measures. A synthetic signal is indicated by a "No" in the Measured column.

\begin{tabular}{|c|c|c|c|c|}
\hline Type & Measured & Signal & Standard & Characteristics \\
\hline I & Yes & 8-PSK & Stanag 4285 & $\begin{array}{l}75 \text { baud long interleaving } \\
\text { Sub-Carrier } 1800 \mathrm{~Hz} \\
\text { Bandwidth } 600-3000 \mathrm{~Hz}\end{array}$ \\
\hline II & Yes & FSK Alt. Wide & $\begin{array}{l}\text { Mil-Std-188-110A } \\
\quad \text { Sec. } 5.1 .1\end{array}$ & $\begin{array}{l}75 \text { baud no interleaving } \\
\text { Mark:1915 Hz Space:2085 Hz }\end{array}$ \\
\hline III & Yes & FSK Wide & $\begin{array}{l}\text { Mil-Std-188-110A } \\
\text { Sec. } 5.1 .2\end{array}$ & $\begin{array}{l}75 \text { baud no interleaving } \\
\text { Mark:1575 Hz Space: } 2425 \mathrm{~Hz}\end{array}$ \\
\hline IV & Yes & FSK Narrow & $\begin{array}{l}\text { Mil-Std-188-110A } \\
\text { Sec. } 5.1 .3\end{array}$ & $\begin{array}{l}75 \text { baud no interleaving } \\
\text { Mark: } 2762.5 \mathrm{~Hz} \text { Space: } 2847.5 \mathrm{~Hz}\end{array}$ \\
\hline $\mathrm{V}$ & No & 2-FSK & - & $\begin{array}{l}\text { Continuous-phase FSK } \\
150 \text { baud - Sub-Carrier } 2000 \mathrm{~Hz} \\
\text { Mark: } 1915 \mathrm{~Hz} \text { Space:2085 Hz }\end{array}$ \\
\hline VI & No & 2-PSK & - & 150 baud - Sub-Carrier $2000 \mathrm{~Hz}$ \\
\hline VII & No & 8-PSK & Stanag 4285 & $\begin{array}{l}1200 \text { baud } \\
\text { Sub-Carrier } 1800 \mathrm{~Hz} \\
\text { Bandwidth } 600-3000 \mathrm{~Hz}\end{array}$ \\
\hline
\end{tabular}
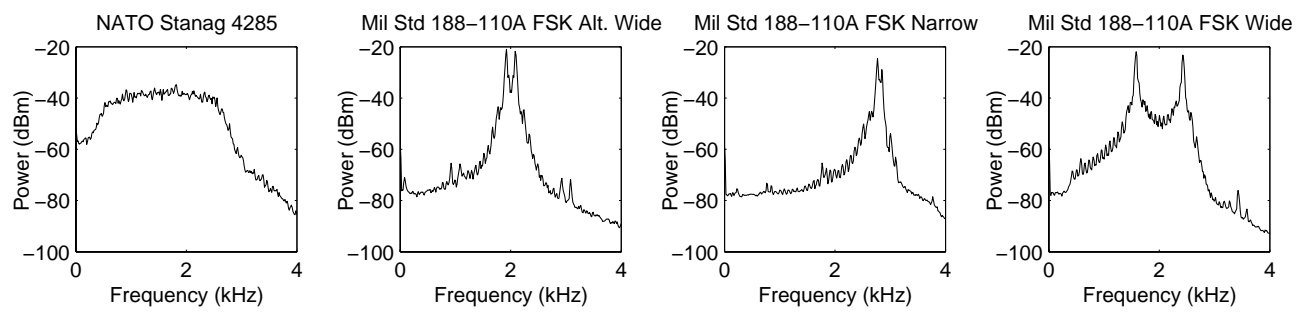

Figure 2. Power-spectra of various real HF signals (far-left: Type I signal; center-left: Type II signal; center-right: Type IV signal; far-right: Type III signal; — see Table 1).

Assume that a baseband HF signal is digitized by a $Q$-level quantizer. The $Q$ levels correspond to an alphabet of $Q$ symbols. For example, if $Q$ is 256 the alphabet may consist of hexadecimal values $\$ 00$ to $\$ F F$. With this in mind, two experiments are conducted to investigate the usefulness of entropic distance for modulation recognition. The first studies the effect that quantization level has on entropic distance. The second investigates the ability of entropic distance to separate signals.

For the first experiment synthetic HF signals from Table 1 are digitized with a $Q$-level quantizer. For each trial, the unknown received signal, $\boldsymbol{A}$, takes on one of these synthetic signals while the reference signal, $\boldsymbol{B}$, is a random selection of symbols from the entire $Q$-symbol alphabet. Then entropic distance is computed between each $\boldsymbol{A}$ and $\boldsymbol{B}$ for varying $Q$ and varying length of $A$. The second experiment investigates entropic distance between real signals from Table 1 and random selections of symbols from the entire $Q$-symbol alphabet. It does 
this by computing the mean of a number of trials for various random segments of $\boldsymbol{A}$ and $\boldsymbol{B}$. For the purposes of computing Equation (6), either 12-bit LZW compression or Zip 2.3 compression is used in these experiments.

\subsection{Measurement of HF Noise}

Data from each DDC sweep is segmented into bands where the noise statistics are assumed stationary in the wide sense. Given the sweep rate, $F_{\mathrm{swp}}$, and the sampling rate, $F_{\mathrm{s}}$, the bandwidth covered by the sweep at each sampling instant is denoted by $f_{\mathrm{n}}=\frac{F_{\mathrm{swp}}}{F_{\mathrm{s}}}$. Therefore the number of samples in a given bandwidth, BW, is $N_{\text {samp }}=\frac{\mathrm{BW}}{f_{\mathrm{n}}}$. Within $\mathrm{BW}, M$ subchannels of bandwidth, $\mathrm{BW}_{\mathrm{c}}$, are chosen such that $M=\frac{\mathrm{BW}}{\mathrm{BW}_{\mathrm{c}}}$ and where the number of samples in each subchannel are $L=\frac{\mathrm{BW}_{\mathrm{c}}}{f_{\mathrm{n}}}$.

The $90^{t h}$ percentile of the absolute value of the samples in each subchannel of the segment is taken as a measure of the signal level in each subchannel. From the list of signal levels for each subchannel a percentage are chosen. All the time-series samples that correspond to the subchannels falling below an $L_{p}$ percent threshold are considered noise samples. The HF noise probability density function is computed based on these samples.

\section{RESULTS}

\subsection{Entropy Results}

The $Q$ levels of the quantizer correspond to an alphabet of $Q$ symbols with a dynamic range of $20 \log _{10} Q$. For example, if $Q=2^{n}$ and $n=16$ bits then the dynamic range is $96 \mathrm{~dB}$. Consequently, we expect that if the received signal level stays the same and if the size of the alphabet increases then the total entropy should also increase. Why?

Consider that (6) is based on the compression of segments $A+a, A+b, B+a, B+b, A$, and $B$ where $a$ and $b$ are small segements of $\boldsymbol{A}$ and $\boldsymbol{B}$ respectively. As the compressor sequentially processes $A+b$ it begins by creating a dictionary of symbols present in $A$. The symbols of $A$ are already present in $B$, since $B$ consists of symbols from the entire $Q$-symbol alphabet, but the symbols of $A$ may not span the whole range of the alphabet. For example, a $1 \mathrm{~V}$ signal quantized by an analog-to-digital converter (ADC) with a full-scale input range of $2 \mathrm{~V}$ would only span half the symbols in the $Q$-symbol alphabet. Eventually, the compressor reaches the end of $A$ in the $A+b$ sequence and begins compressing $b$. The data segment, $b$, may well span the entire $Q$-symbol alphabet since $b$ came from $\boldsymbol{B}$. As a result, the compressor sees new symbols that are not already present in its dictionary formed on $A$ and therefore is forced to create new dictionary entries. This causes the efficiency of the compressor to decrease and therefore the compressed length of $A+b$ is higher than it would be if $A$ also contained the entire $Q$-symbol alphabet. A similar argument goes for $B+a$ but does not hold for $A+a, B+b, A$, or $B$ because for these sequences the compressor dictionary consists of symbols entirely from $\boldsymbol{A}$ or $\boldsymbol{B}$.

Figure 3 does indeed reflect this logic by showing that for a synthetic Stanag 4285 signal the relative entropy measure, as computed by (4), increases as $Q$ increases.

Next, $\boldsymbol{A}$ is allowed to take on any of the synthetic signals in Table 1 and the effect on entropic distance is observed. Figure 4 shows that (6) provides separation of signals for sequence lengths greater than about 4,000. The separation between modulations also increases with $Q$. For an 8-bit quantizer the alphabet is quite coarse compared to the 16-bit quantizer. Note that the entropic distance briefly exceeds Shannon's entropy ${ }^{5}$ for the case where the quantizer has 16-bits. It is likely that relatively few samples at the point of overshoot causes the entropy measure to be more sensitive to the noise signal $B$. So, the quantization level does affect the entropy measure when computed using Benedetto's compression method.

Shannon ${ }^{14}$ showed that the computation of true entropy relied on the independence of successive symbols. That is, if successive symbols (in this case quantized samples) are not independent then conditional entropies must be used to determine the true entropy. For the synthetic signals just presented the successive samples are assumed independent even though, strictly speaking, they are not independent. They are not independent because successive samples can be predicted with some certainty based on foreknowledge of the signal waveform. However, successive samples from real HF signals have a larger degree of independence because of the nature of HF communications, which encompasses impulsive HF noise, fading, multipath, and Doppler spreading; and, the receiver is not necessarily aware of the type of incoming signal. 


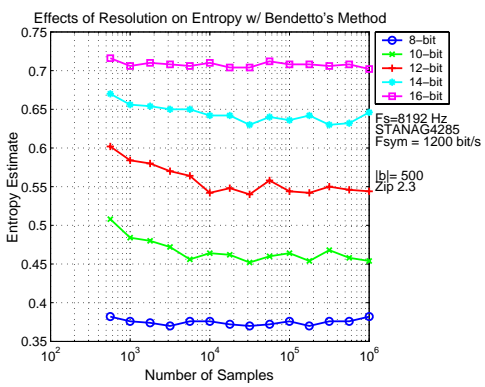

Figure 3. Effect of quantizer level, $Q$, on relative entropy for varying lengths of $A$. The length of $b$ is constant at 500 symbols. Here $B$ is a random selection of symbols from the $Q$-symbol alphabet and $A$ is a synthetic 8 -PSK signal (Stanag 4285 carrying a random message at $1200 \mathrm{bit} / \mathrm{s}$ ) digitized with a $Q$-level quantizer. Zip 2.3 compression is used.
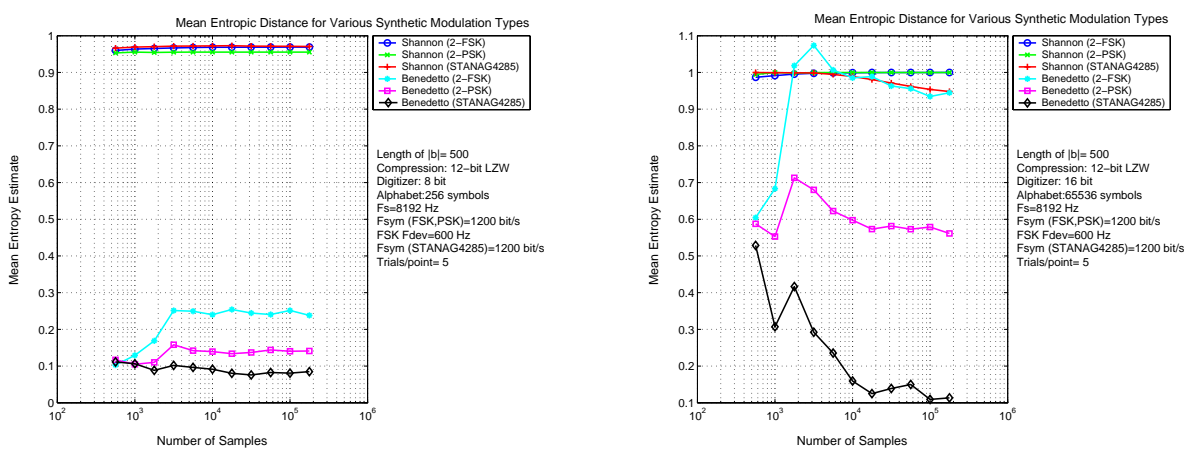

Figure 4. Effect of quantizer level, $Q$ (left: $Q=256$, right: $Q=65,536$ ), on relative entropy for varying lengths of $A$. The length of $b$ is constant at 500 symbols. Here $B$ is a random selection of symbols from the $Q$-symbol alphabet and $A$ is a synthetic 2-FSK, 2-PSK, or 8-PSK signal (Stanag 4285) digitized with a $Q$-level quantizer. In all cases the synthetic signals carry a random message at $1200 \mathrm{bit} / \mathrm{s}$ and 12-bit LZW compression is used. Entropy as computed by Shannon's $\operatorname{method}^{5}$ is also included for reference.
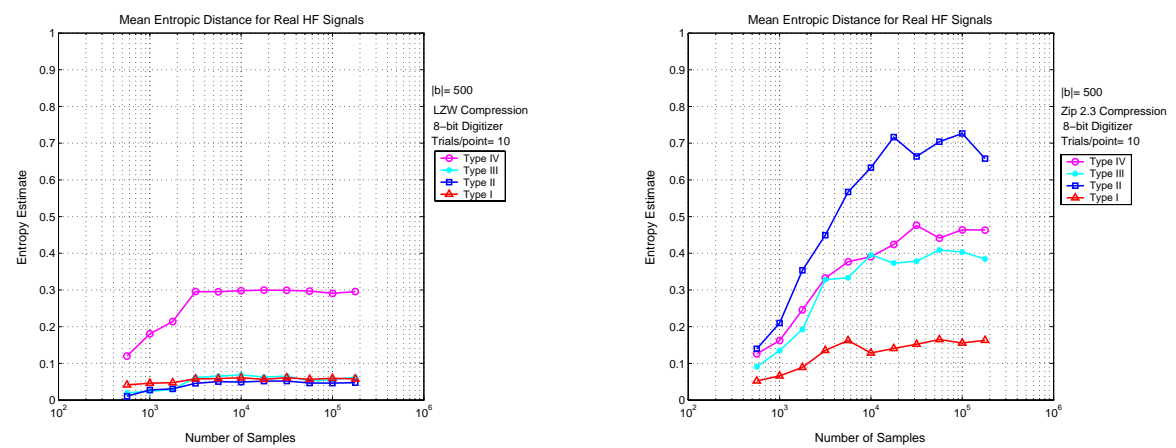

Figure 5. Entropic distance between Type I - IV HF signals and random sequences of the $Q$-symbol $(Q=256)$ alphabet for varying lengths of $A$. The length of $b$ is constant at 500 symbols. Here $\boldsymbol{B}$ is a random selection of symbols from the $Q$-symbol alphabet and $\boldsymbol{A}$ is a real HF groundwave signal digitized with the equivalent of an $\approx 8.5$-bit quantizer. Each point is the mean of ten (10) trials. In all cases the real signals carry a random message. Note that the entropic distance computed using LZW compression and a 256-symbol alphabet provides little separation (left), yet entropic distance computed using Zip 2.3 compression provides good separation (right). The standard deviation at each point is negligible for both plots. 

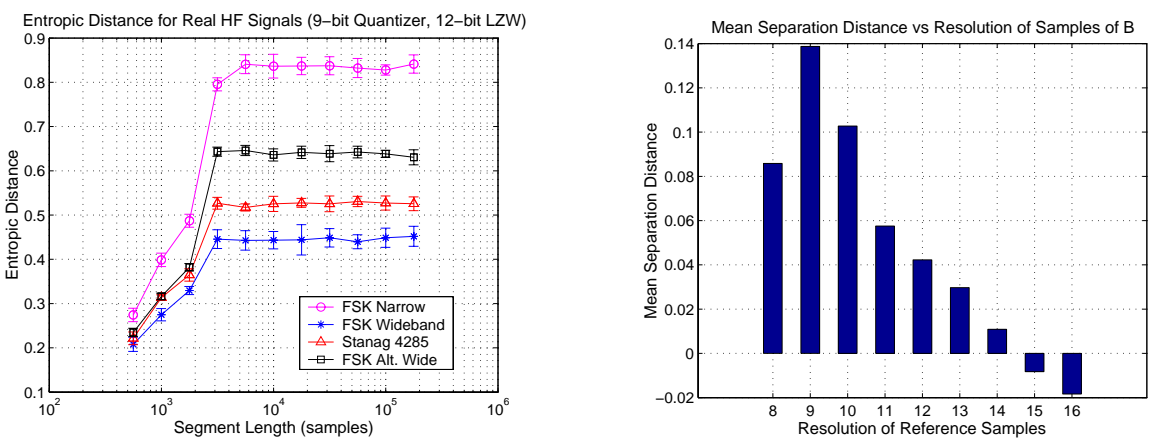

Figure 6. Compared with Figure 5, entropic distance between Type I - IV HF signals (with $\approx 8.5$-bit resolution) and random sequences with a $Q$-symbol alphabet $(Q=512)$ provides good separation (left). Again, the length of $b$ is constant at 500 symbols, each point is the mean of ten (10) trials (error bars show the standard deviation at each sample point), and the real signals carry a random message. Also plotted is the mean separation distance between entropy curves for various alphabet sizes for $\boldsymbol{B}($ right). It is clear that increasingly large alphabets decrease the mean separation distance. Negative separation distances indicate that the entropy curves overlap.

Application of the entropic distance measurement method to real HF groundwaves results in characteristics similar to those of Figure 4. Figure 5 shows that entropy computed using 12-bit LZW compression and an 8-bit alphabet provides little discernable separation of the signals. Entropy computed using Zip 2.3 compression shows good separation of the signals. One might suggest using a larger $Q$-symbol alphabet with 12-bit LZW compression but this does not necessarily improve the separation of signals.

To determine an appropriate alphabet size, we define the mean separation distance (MSD) as a measure of the empty space between entropy curves. A large positive MSD indicates that the curves are sufficiently separable. A low or negative MSD indicates that the curves generally overlap and are not easily separable. Let $S_{i j}$ be the random variable representing all trials at the $j^{\text {th }}$ plotting point for the $i^{\text {th }}$ entropy curve, and let $\vec{S}_{i}$ represent a vector of averages for the $i^{\text {th }}$ curve such that $\vec{S}_{i}=\left[\mathrm{E}\left(S_{i 1}\right) \mathrm{E}\left(S_{i 2}\right) \mathrm{E}\left(S_{i 3}\right) \cdots \mathrm{E}\left(S_{i j}\right)\right]$, where $\mathrm{E}(\cdot)$ is the expectation operator. Similarly, the standard deviation vector is, $\overrightarrow{\sigma_{i}}=\left[\begin{array}{lllll}\sigma\left(S_{i 1}\right) & \sigma\left(S_{i 2}\right) & \sigma\left(S_{i 3}\right) & \cdots & \sigma\left(S_{i j}\right)\end{array}\right]$, where $\sigma(\cdot)$ is the standard deviation function. Then the mean separation distance is

$$
\operatorname{MSD}=\mathrm{E}\left(\frac{2 !(N-2) !}{N !} \sum_{i=0}^{N-2} \sum_{j=i+1}^{N-1}\left\|\overrightarrow{S_{i}}-\overrightarrow{S_{j}}\right\|-\left\|\overrightarrow{\sigma_{i}}-\overrightarrow{\sigma_{j}}\right\|\right),
$$

where $\|\cdot\|$ is the Euclidean distance operator and $N$ is the number of entropy curves being compared.

Equation (7) is plotted in Figure 6 and shows that a 512-symbol (9-bit quantizer) alphabet is near optimal for the real signal set in Table 1. Larger or smaller alphabet sizes for $\boldsymbol{B}$ actually decrease the mean separation distance between signals. Therefore, the most appropriate quantizer to use with the LZW algorithm is one that has a resolution similar to or slightly larger than the resolution of the real signal. This is necessary so that the dictionary in the LZW algorithm does not become so large that the effect of $b$ goes unnoticed. However, note that the 8-bit alphabet also shows a relatively high MSD. This is a result of the large separation between the Type IV signal (see Figure 5) and the other real signals, for which there is little separation. Clearly, the MSD cannot be used exclusively to choose an alphabet size but must be used in conjunction with an entropy plot (e.g. Figures 5 and 6$)$.

\subsection{HF Noise Results}

The noise in the HF spectrum comprises multiple components: galactic noise, atmospheric noise, and manmade noise. Galactic noise is produced by cosmic radiators such as the sun and other stars. Atmospheric noise changes with time of year and is largely influenced by lightning activity, while man-made noise is produced by electro-magnetic radiating devices (e.g. electric machinery, computers). 
Prior to sunrise, the D-region of the ionosphere does not exist and therefore signals from distant emitters (transmitters and noise sources) add to the noise level in the HF band. During the period just after sunrise the D-region of the ionosphere builds and begins absorbing much of the distant man-made HF interference.

Consider Figure 7 which shows the PDF of HF noise between $6.1 \mathrm{MHz}$ and $6.3 \mathrm{MHz}$ for various times shortly after sunrise (6:51 am local time (LT) on February 17, 2005) at a site with relatively high man-made noise levels in Adelaide, Australia. The subchannel bandwidth, $\mathrm{BW}_{\mathrm{c}}$, is $1.2 \mathrm{kHz}$ and the bandwidth over which the noise statistics are assumed stationary, BW, is $228 \mathrm{kHz}$ (these choices of bandwidth are reasonable for the purpose of this demonstration). From 7:00 am to 8:00 am the PDFs are decidely non-Gaussian because most noise emitting sources have not yet been turned on (people are just waking). During this time the D-region is becoming more ionized and is absorbing sky-wave transmissions. After 8:00 am the PDF becomes more and more Gaussian ${ }^{\ddagger}$.

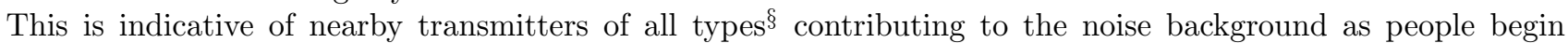
enabling their devices. Consequently, man-made noise through ground-wave transmissions are likely affecting the HF noise PDF after sunrise despite the fact that the D-region is absorbing most distant man-made noise transmissions.

Though not shown, similar results are achieved for the PDF at frequencies between $11.6 \mathrm{MHz}$ and $11.8 \mathrm{MHz}$, as well as between $13.6 \mathrm{MHz}$ and $13.9 \mathrm{MHz}$. At the "noisy" measurement site, the PDF at frequencies between 5.4 MHz and 5.6 MHz is markedly Gaussian at all times. This band is highly used for each hour of the day.

Though the results just presented are not conclusive proof that the HF noise PDF is non-Gaussian, it does show that depending on the site, the time of day, and the frequency the assumption of Gaussian noise that many researchers make for their analyses can be quite inaccurate. Consequently any HF communications model must account for the surrounding HF environment (i.e. mixture and strength of noise components), the frequency band, and the time of day. Given the enormous difficulties involved in developing modulation recognition algorithms, it is not unreasonable therefore to begin with an assumption of Gaussian noise and then to progress to a more realistic form of noise.

So, if the HF noise PDF is non-Gaussian what distribution does it resemble? It actually resembles the BiKappa distribution addressed by Leubner and Vörös. ${ }^{15}$ This distribution is very similar to the HF noise PDF as described by Johnson. ${ }^{16}$ Figure 8 illustrates the fit of the Bi-Kappa distribution to two PDFs of Figure 7.

\section{FUTURE WORK}

Coherence, as defined above, is not practical for identifying unknown signal types. Entropy, as defined above, does separate contrived and real signals. The next step in the research is to focus more on the method of feature extraction. Now a brief review of research literature is necessary to set the context of the future work and to highlight other parameters that may prove useful in modulation recognition for HF signals.

Aisbett ${ }^{17}$ points to a potentially good technique for SNR measurements, discrimination of constant envelope signals versus varying envelope signals, as well as the detection of the number of states of multi-level AM. The function, called \#(X,Y), is the product of the means of signals $X$ and $Y$ less their covariance. It appears able to discriminate contrived signals and can separate CW, AM, and FM signals. However, the main problem with this work is the common assumption of Gaussian noise. As we have seen, HF noise cannot be assumed to be Gaussian.

In his detection method for multi-carrier modulations, Akmouche ${ }^{18}$ shows that a modified kurtosis function provides some separation of BPSK, QPSK, 16-/256-QAM and OFDM-32 on synthetic data with an SNR of $0 \mathrm{~dB}$. The modified kurtosis appears useful in providing a clear distinction between OFDM and single carrier modulations. Again, Gaussian noise is assumed. Moreover the modified kurtosis is not generally applicable to cyclostationary signals, though this is not clear in the paper. And finally, his modified kurtosis is sensitive to noise and the number of samples on which the modified kurtosis is computed. Thus the apparent advantage of the modified kurtosis for real signals is in the discrimination of multi-carrier modulations from single-carrier

\footnotetext{
${ }^{\ddagger}$ This appears to be a result of the central limit theorem.

$\S$ These transmitters could be genuine HF transceivers, or welders and car ignitions for example.

๑ Multi-tone PSK is a common HF signal.
} 

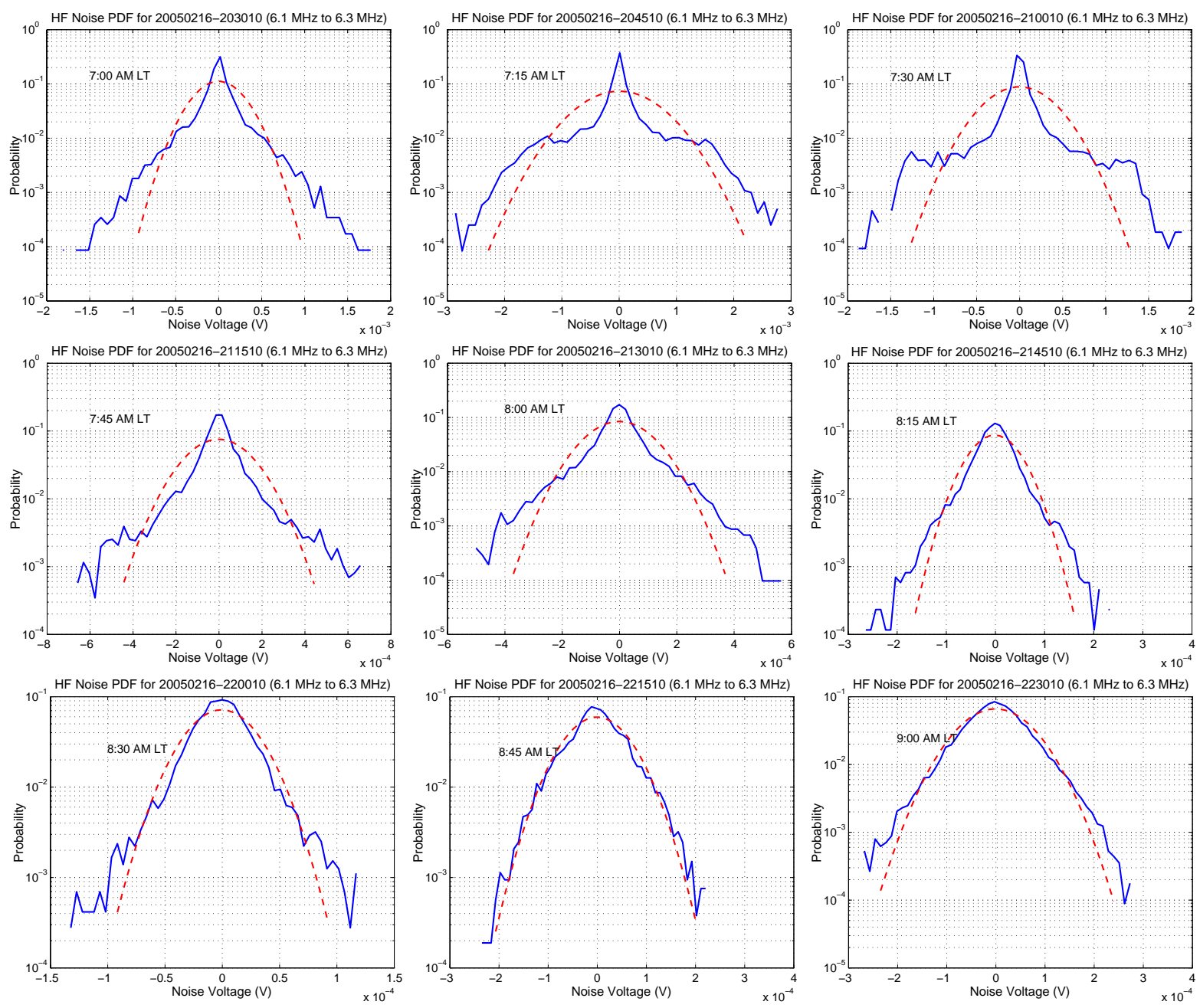

Figure 7. PDF of HF noise for various times shortly after sunrise, 6:51 am local time (LT), from $6.1 \mathrm{MHz}$ to $6.3 \mathrm{MHz}$. A Gaussian PDF is superimposed (dashed curves) to show how poorly a Gaussian PDF fits the PDF of HF noise. Notice that as the day progresses the PDF becomes more and more Gaussian. This is indicative of transmitters of all types contributing to the noise background as people awake and begin enabling their devices. These devices could be genuine HF transceivers, or welders and car ignitions. The central-limit theorem seems to be playing a role here.
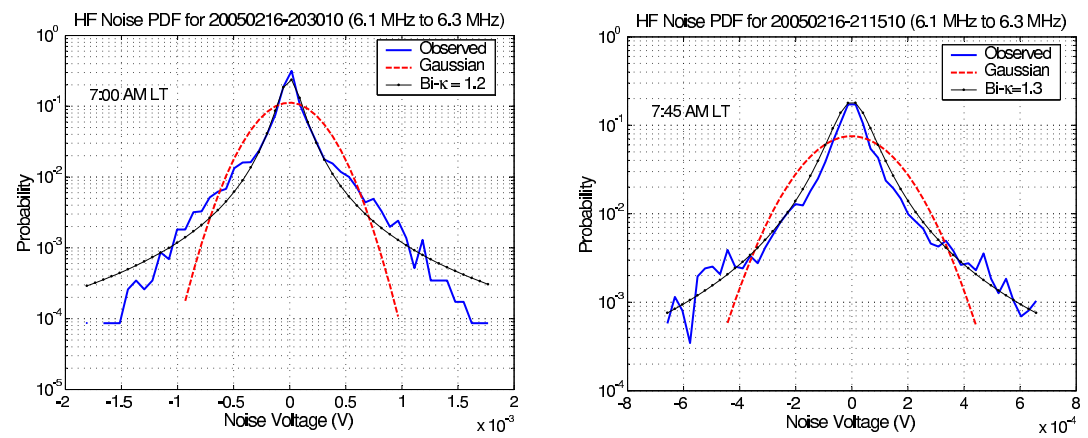

Figure 8. Fit of a Bi-Kappa distribution to the PDF of HF noise at 7:00 am (left) and 7:45 am (right). Compare the peak and tails of the Bi-Kappa distribution (dotted curve) to the Gaussian distribution (dashed curve). 
modulations. Since the modified kurtosis is sensitive to noise, real signals may require noise removal prior to computation of the modified kurtosis. One method for noise removal is based on the wavelet transform.

Hsue et $a l^{19}$ describe a simple zero-crossing method that separates synthetic CW, m-ary PSK, and m-ary FSK signals. Carrier frequency, carrier-to-noise ratio (CNR), and symbol rate can also be estimated using zero-crossings. Classification of the signal type is made using histograms. The disadvantages of Hsue's work is that Gaussian noise is assumed, the method applies only to constant envelope signals, and the CNR must be greater than $15 \mathrm{~dB}$ to be effective on synthetic signals. For practical use the CNR will need to be much higher. The zero-crossing method and parameter extraction functions are attractive because of their simplicity but appear unsuitable for real signals that are noisy. Nevertheless, Hsue's classification method appears suitable for classifying real signals. His classification method consists of a decision tree and pattern recognition algorithm.

Others have taken a hybrid approach to modulation recognition. Nandi, Azzouz, and Wong ${ }^{20-22}$ describe a feature extraction and classification algorithm for separating m-ary ASK, m-ary FSK, m-ary PSK, AM, FM, mary QAM, and V.29 and V.32 (modem modulation standards). Feature extraction is obtained through statistical methods, while classification is based on neural networks. Without noise and with suitable training, their method is capable of recognizing the modulation types with no error. With signal-to-noise ratios (SNRs) of $15 \mathrm{~dB}$ and $20 \mathrm{~dB}$, their algorithm is able to recognize the modulation types with less than $10 \%$ error. The authors, however, do not mention the noise model for their simulations, do not consider the effects of real noisell, multi-path, or Doppler shifts on the performance of the system. All of these strongly influence HF communications and so it is doubtful that the performance figures they quote are achievable for practical HF transmissions. Furthermore, their methods require oversampling of the signal by as much as eight times, which is often not desirable in real-time direct down-conversion systems or software radio systems where the HF signal is sampled directly.

Unlike many others, Ketterer $e t a l^{23}$ do apply their algorithms to real signals. They use the cross-MargenauHill distribution (CMHD), which is related to cross-correlation and convolution, as a feature function for identifying m-ary PSK and its modulation level. An auto-regressive (AR) covariance method is used for accentuating the symbol frequencies of m-ary FSK while the variance of the signal envelope is used to identify amplitude modulated signals (e.g. ASK, QAM). The disadvantages of the methods are that the SNR must be greater than $10 \mathrm{~dB}$, and an accurate estimation of the signal's center frequency is critical for determining the modulation level of m-ary PSK. Neverthless, the CMHD is a potentially useful method for PSK and FSK signal identification.

With this wealth of research in mind, the future work focuses on creating, for an unknown signal, a feature vector consisting of the following parameters: center frequency, bandwidth, signal-to-noise ratio (SNR), signal envelope, symbol frequencies, cross-Margenau-Hill distribution, kurtosis, signal constellation, signal powerspectral-density (PSD), modulation level, auto-regressive covariance, and entropic distance. These parameters can be obtained using methods discussed above, but we intend on extracting this information from the outputs of a set of parallel algorithms in the time-frequency space, namely, the wavelet transform, the short-time-Fourier transform, the complete Fourier transform, and a number of time-domain processing methods. These parameters will be passed to a simple classification method. The classification method is likely to be based on a majority logic and confidence level decision boundary.

\section{SUMMARY}

A brief review has been presented of the usefulness of the coherence function and entropy measure for modulation recognition of real HF signals. It is shown that the coherence function is impractical and that the entropy measure using compression techniques is feasible. It is also demonstrated that the probability-density-function (PDF) of HF noise is not necessarily Gaussian and in fact depends on geographic location, time of day, and surroundings (among other things). Also, a path toward the development of a robust system for automatically recognizing unknown HF signals is described.

\section{ACKNOWLEDGMENTS}

Data for noise analysis is provided by Nigel Brine (School of Electrical \& Electronic Engineering, The University of Adelaide). Financial support for this work is provided by the The University of Adelaide and Ebor Computing.

\footnotetext{
"An SNR of less than $15 \mathrm{~dB}$ is quite common for radio communications.
} 


\section{REFERENCES}

1. Nobel Lectures - Physics 1901-1921, Elsevier Publishing Company, Amsterdam, 1967.

2. J. Smith, Modern Communication Circuits, McGraw-Hill Series in Electrical Engineering, McGraw-Hill Inc., New York, 1st ed., 1986. pp. 1-13.

3. J. E. Giesbrecht, R. Clarke, and D. Abbott, "Improved techniques for monitoring the HF spectrum," in Proceedings of SPIE, 5274, pp. 112-122, 2004.

4. J. E. Giesbrecht, R. Clarke, and D. Abbott, "Monitoring the HF spectrum in the presence of noise," in Proceedings of SPIE, 5473, pp. 76-84, 2004.

5. J. E. Giesbrecht, R. Clarke, and D. Abbott, "Modulation recognition for HF signals," in Proceedings of SPIE, 5649, pp. 501-512, 2005.

6. J. E. Giesbrecht, R. Clarke, and D. Abbott, "Coherence as a feature of real HF signals," in Proceedings of SPIE, 5847, pp. 188-198, 2005.

7. D. Benedetto, E. Caglioti, and V. Loreto, "Language trees and zipping," Physical Review Letters 88(4), 2002. 048702.

8. D. Landgrebe, "On Information Extraction Principles for Hyperspectral Data," tech. rep., School of Electrical and Computer Engineering, Purdue University, West Lafayette, IN, USA, 47907-1285, 1997.

9. J. Ma, J. Theiler, and S. Perkins, "Two realizations of a general feature extraction framework," Pattern Recognition 37, pp. 875-887, 2004.

10. B.-C. Kuo and D. A. Landgrebe, "Nonparametric Weighted Feature Extraction for Classification," IEEE Transactions on Geoscience and Remote Sensing 42(5), pp. 1096-1105, 2004.

11. X. Wang and K. K. Paliwal, "Feature extraction and dimensionality reduction algorithms and their applications in vowel recognition," Pattern Recognition 36, pp. 2429-2439, 2003.

12. C. Distante, M. Leo, P. Siciliano, and K. C. Persaud, "On the study of feature extraction methods for an electronic nose," Sensors and Actuators B 87, pp. 274-288, 2002.

13. N. L. Brine, C. C. Lim, A. D. Massie, and W. Marwood, "A pre-emptive null-steering ionosonde," Fourth Symposium of Radiolocations and Direction Finding, Southwest Research Institute, (San Antonio, Texas), 2002.

14. C. E. Shannon, "A Mathematical Theory of Communication," Bell System Technical Journal 27, pp. 379423, 623-656, July, October 1948.

15. M. P. Leubner and Z. Vörös, "A nonextensive entropy path to probability distributions in solar wind turbulence," Nonlinear Processes in Geophysics 12, pp. 171-180, 2005.

16. E. E. Johnson, R. I. Desourdis Jr., G. D. Earle, S. C. Cook, and J. C. Ostergaard, Advanced High-Frequency Radio Communications, Artech House Inc., 685 Canton St., Norwood MA, USA, 02062, 1997.

17. J. Aisbett, "Automatic Modulation Recognition Using Time Domain Parameters," Tech. Rep. ERL-0367TR, Electronic Research Laboratory DSTO, Department of Defence, Commonwealth Government of Australia, November 1986.

18. W. Akmouche, "Detection of multicarrier modulations using 4th-order cumulants," in MILCOM 1999 Military Communications Conference, 1, pp. 432-436, Institution of Electrical and Electronic Engineers, October 1999.

19. S.-Z. Hsue and S. S. Soliman, "Automatic modulation classification using zero crossing," IEE Proceedings 137, pp. 459-464, December 1990.

20. A. K. Nandi and E. E. Azzouz, "Modulation recognition using artificial neural networks," Signal Processing 56, pp. 165-175, 1997.

21. A. K. Nandi and E. E. Azzouz, "Algorithms for Automatic Modulation Recognition of Communication Signals," IEEE Transactions on Communications 46, pp. 431-435, April 1998.

22. M. L. D. Wong and A. K. Nandi, "Automatic digital modulation recognition using spectral and statistical features with multi-layer perceptrons," in Proceedings of the IEEE International Symposium on Signal Processing and its Applications, 2, pp. 390-393, (Kuala Lumpur, Malaysia), 2001.

23. H. Ketterer, F. Jondral, and A. H. Costa, "Classification of modulation models using time-frequency methods," in Proceedings of the IEEE 1999 International Conference on Acoustics, Speech, and Signal Processing, 5, pp. 2471-2474, (Phoenix, Arizona), 1999. 